\title{
EVALUATION OF PROMPT FISSION GAMMA RAYS FOR USE IN SIMULATING NUCLEAR SAFEGUARD MEASUREMENTS
}

Timothy E. Valentine

October 1999

\author{
Prepared by \\ OAK RIDGE NATIONAL LABORATORY \\ Oak Ridge, Tennessee 37831-6004 \\ managed by \\ LOCKHEED MARTIN ENERGY RESEARCH CORP. \\ for the \\ U.S. DEPARTMENT OF ENERGY \\ under contract DE-AC05-96OR22464
}


This page intentionally left blank. 


\section{CONTENTS}

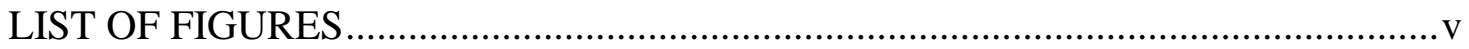

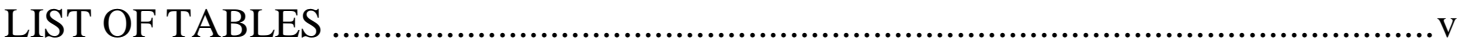

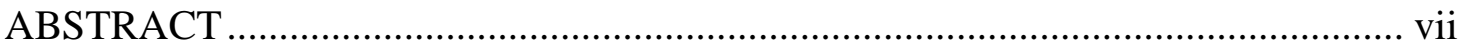

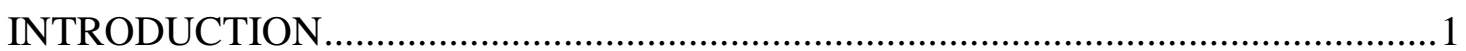

TOTAL PROMPT GAMMA RAY ENERGY FROM FISSION ...............................

AVERAGE NUMBER OF GAMMA RAYS FROM FISSION .................................

MULTIPLICITY DISTRIBUTION OF GAMMA RAYS FROM FISSION ................4

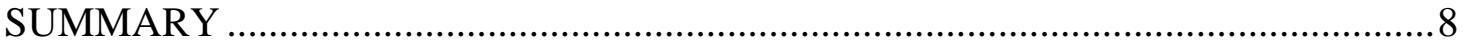

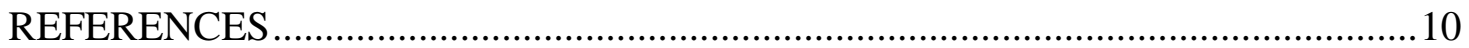


This page intentionally left blank. 


\section{LIST OF FIGURES}

Figure 1 Gamma ray probability distribution function for spontaneous fission of ${ }^{252} \mathrm{Cf}$ from Eq. 6 with parameters $C_{1}=0.675, C_{2}=6.78$,

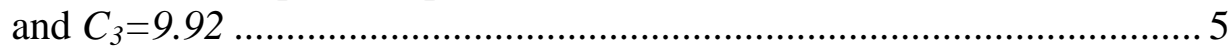

Figure 2 Comparison of Brunson's model with parameters $C_{1}=0.675, C_{2}=6.78$, and $C_{3}=9.92$ for ${ }^{252} \mathrm{Cf}$ with the negative binomial distribution .. 8

\section{LIST OF TABLES}

Table 1 Measured properties of gamma rays from fission ................................ 2

Table 2 Estimated properties of gamma rays from spontaneous fission of ${ }^{238} \mathrm{U}$, ${ }^{238} \mathrm{Pu},{ }^{240} \mathrm{Pu},{ }^{242} \mathrm{Pu},{ }^{242} \mathrm{Cm}$, and ${ }^{244} \mathrm{Cm}$ using Eqs. 2, 4, and 5 .................. 4 
This page intentionally left blank. 


\begin{abstract}
Nondestructive assay methods that rely on measurement of correlated gamma rays from fission have been proposed as a means to determine the mass of fissile materials. Sensitivity studies for such measurements will require knowledge of the multiplicity of prompt gamma rays from fission; however, a very limited number of multiplicity distributions have been measured. A method is proposed to estimate the average number of gamma rays from any fission process by using the correlation of neutron and gamma emission in fission. Using this method, models for the total prompt gamma ray energy from fission adequately reproduce the measured value for thermal neutron induced fission of ${ }^{233} \mathrm{U}$. Likewise, the average energy of prompt gamma rays from fission has been adequately estimated using a simple linear model. Additionally, a method to estimate the multiplicity distribution of prompt gamma rays from fission is proposed based on a measured distribution for ${ }^{252} \mathrm{Cf}$. These methods are only approximate at best and should only be used for sensitivity studies. Measurements of the multiplicity distribution of prompt gamma rays from fission should be performed to determine the adequacy of the models proposed in this article.
\end{abstract}


This page intentionally left blank. 


\section{INTRODUCTION}

Standard nondestructive assay measurement methods use the emission of prompt neutrons as one means to determine the mass of fissile materials that contain spontaneous fission nuclides such as ${ }^{240} \mathrm{Pu} .{ }^{1,2}$ Recently, extensions of nondestructive assay measurements using detectors sensitive to both neutrons and gamma rays have been proposed. ${ }^{3}$ The gamma ray multiplicity data must be available to determine the sensitivity of such measurements to fissile mass. Ideally, measurements of the multiplicity distribution should be performed to determine the gamma ray multiplicity data; however, such measurements have not been performed for many spontaneous fission nuclides. Therefore, an approximate method to estimate the gamma ray multiplicity data has been devised based on the average number of gamma rays from fission. In some cases, the average number of prompt gamma rays from fission has not been measured and an approximate method to estimate it has been provided.

This brief paper describes the methodology used to estimate the total prompt gamma ray energy from fission in Section 2 and to estimate the average number of prompt gamma rays from fission in Section 3. A description of the estimated probability distribution data is provided in Section 4. Finally, a summary of this work is provided in Section 5.

\section{TOTAL PROMPT GAMMA RAY ENERGY FROM FISSION}

The correlation between the total prompt gamma ray energy from fission and the number of neutrons emitted from fission is used to estimate the average number of gamma rays from fission in lieu of performing a measurement. Competition in the emission of prompt gamma rays and neutrons from the de-excitation of fission fragments has been observed experimentally. This competition is expected due to the quantum mechanical selection

rules for conservation of angular momentum. ${ }^{4}$ Gamma rays are primarily responsible for decreasing the angular momentum of the excited fission fragments. Nifenecker et al. ${ }^{5}$ demonstrated that a linear relationship exists between the total prompt gamma ray energy, $E_{t}$, from fission and the number of prompt neutrons, $v$, from the spontaneous fission of ${ }^{252} \mathrm{Cf}$ :

$$
E_{t}(v)=0.75 v+4.0
$$

Sufficient experimental data exist to determine if the relationship in Eq. 1 is applicable for all fission processes. For example, the measurements of Pleasonton et al. ${ }^{6}$ show a strong dependence of the total prompt gamma ray energy on the fragment mass. Because the fragment mass yield curve is dependent on the original fission nuclide, we postulate that the total gamma ray energy is a function of both the pre-fission mass and the number of neutrons emitted from fission. Hence, a more general extension of Eq. 1 would relate the total prompt gamma ray energy, $E_{t}(v, Z, A$, $)$, to the pre-fission mass $(A)$ and the number of neutrons from fission via 


$$
E_{t}(v, Z, A)=\varphi(Z, A) v+4.0
$$

The function $\varphi(Z, A)$ is an arbitrary function determined by trial and error to provide the most linear relationship between the estimated values of $E_{t}$ and the average measured values for ${ }^{235} \mathrm{U},{ }^{239} \mathrm{Pu}$, and ${ }^{252} \mathrm{Cf}$. A summary of the measured properties of prompt gamma rays from thermal-neutron induced and spontaneous fission for several nuclides is presented in Table 1.

Table 1. Measured properties of gamma rays from fission

\begin{tabular}{|c|c|c|c|c|}
\hline Isotope & $\begin{array}{l}\text { Total energy } \\
(\mathrm{MeV})\end{array}$ & $\begin{array}{l}\text { Average } \\
\text { number }\end{array}$ & $\begin{array}{c}\text { Average energy } \\
(\mathrm{MeV})\end{array}$ & Reference \\
\hline${ }^{233} \mathrm{U}$ & $6.69 \pm 0.3$ & $6.31 \pm 0.3$ & $1.06 \pm 0.07$ & Pleasonton $^{7}$ \\
\hline \multirow{4}{*}{${ }^{235} \mathrm{U}$} & $6.43 \pm 0.3$ & $6.51 \pm 0.3$ & $0.99 \pm 0.09$ & Pleasonton et al. ${ }^{6}$ \\
\hline & $6.70 \pm 0.4$ & $6.69 \pm 0.3$ & $0.97 \pm 0.05$ & Verbinski et al. $^{8}$ \\
\hline & $7.2 \pm 0.3$ & $7.45 \pm 0.32$ & $0.96 \pm 0.05$ & Pelle and Maienschein ${ }^{9}$ \\
\hline & $6.53 \pm 0.2$ & $6.60 \pm 0.1$ & $0.97 \pm 0.02$ & Average $^{\mathrm{a}}$ \\
\hline \multirow{3}{*}{${ }^{239} \mathrm{Pu}$} & $6.73 \pm 0.35$ & $6.88 \pm 0.35$ & $0.98 \pm 0.07$ & Pleasonton $^{7}$ \\
\hline & $6.82 \pm 0.3$ & $7.23 \pm 0.3$ & $0.94 \pm 0.05$ & Verbinski et al. $^{8}$ \\
\hline & $6.78 \pm 0.1$ & $7.06 \pm 0.2$ & $0.95 \pm 0.03$ & Average \\
\hline \multirow{6}{*}{${ }^{252} \mathrm{Cf}$} & $7.06 \pm 0.35$ & $8.32 \pm 0.4$ & $0.85 \pm 0.06$ & Pleasonton et al. ${ }^{10}$ \\
\hline & $6.84 \pm 0.3$ & $7.80 \pm 0.3$ & $0.88 \pm 0.04$ & Verbinski et al. $^{8}$ \\
\hline & 8.6 & 10 & $0.90 \pm 0.06$ & Bowman and Thompson ${ }^{11}$ \\
\hline & $6.7 \pm 0.4$ & $\mathrm{n} / \mathrm{a}$ & $\mathrm{n} / \mathrm{a}$ & Nardi et al. ${ }^{12}$ \\
\hline & $\mathrm{n} / \mathrm{a}$ & $7.5 \pm 1.5$ & $0.96 \pm 0.08$ & Val'skii et al. $^{13}$ \\
\hline & $6.95 \pm 0.3$ & $7.98 \pm 0.4$ & $0.87 \pm 0.02$ & Average \\
\hline
\end{tabular}

${ }^{a}$ Average quantities are the variance-weighted averages of the measured data of Pleasonton et al. and Verbinski et al.

The data presented in Table 1 were used to determine the function $\varphi(Z, A)$ using the average number of neutrons from fission of ${ }^{235} \mathrm{U},{ }^{239} \mathrm{Pu}$, and ${ }^{252} \mathrm{Cf}$. The average number of prompt neutrons for thermal neutron induced fission of ${ }^{235} \mathrm{U}$ and ${ }^{239} \mathrm{Pu}$ are $2.437 \pm 0.005$ and $2.889 \pm 0.008$, respectively as measured by Gwin et al. ${ }^{14}$ The average number of prompt neutrons from the spontaneous fission of ${ }^{252} \mathrm{Cf}$ is 3.757 as measured by Boldeman and Hines. ${ }^{15}$ The function $\varphi(Z, A)$ was determined by trial and error and varies linearly with $Z^{2} A^{1 / 2}$

$$
\varphi(\mathrm{Z}, \mathrm{A})=2.51( \pm 0.01)-1.13 \cdot 10^{-5}\left( \pm 7.2 \cdot 10^{-8}\right) Z^{2} \sqrt{A} .
$$

No physical significance has yet been attributed to the functional form of $\varphi(Z, A)$. The numbers in parenthesis are the uncertainty in the constant terms obtained by least-square regression assuming no uncertainty in $E_{t}$ or $v$ values used to determine $\varphi(Z, A)$. The 
estimated total prompt gamma ray energy for thermal neutron induced fission of ${ }^{233} \mathrm{U}$ calculated using Eqs. 2 and 3 is $6.61 \pm 0.03 \mathrm{MeV}$ assuming $2.494 \pm 0.006$ neutrons from ${ }^{233} \mathrm{U}$ fission as measured by Gwin et al. ${ }^{14}$ This value is in close agreement with the measured value $(6.69 \pm 0.3 \mathrm{MeV})$ of Pleasonton. This example demonstrates that the total prompt gamma ray energy for spontaneous fission or thermal neutron induced fission can be adequately estimated using Eq. 2 .

\section{AVERAGE NUMBER OF GAMMA RAYS FROM FISSION}

As can be seen from Table 1, the average gamma ray energy varies slightly from one nuclide to another. In the articles by Smith et al. ${ }^{16}$ and by Verbinski et al. ${ }^{8}$ the prompt gamma ray energy spectrum of ${ }^{252} \mathrm{Cf}$ was shown to be softer than the prompt gamma ray spectrum of ${ }^{235} \mathrm{U}$. This would indicate that the average energy of prompt gamma rays from the spontaneous fission of ${ }^{252} \mathrm{Cf}$ would have a lower average value than those from the induced fission of ${ }^{235} \mathrm{U}$. The average prompt gamma ray energy presented in Table 1 decreases essentially linearly with the pre-fission atomic mass. A linear fit of the measured average energy of prompt fission gamma rays from ${ }^{235} \mathrm{U},{ }^{239} \mathrm{Pu}$, and ${ }^{252} \mathrm{Cf}$ produces the following relationship between the average prompt gamma ray energy, the atomic number $(Z)$, and the pre-fission atomic mass $(A)$

$$
<E>=-1.33( \pm 0.05)+119.6( \pm 2.5) Z^{1 / 3} / A \text {. }
$$

The numbers in parenthesis are the uncertainty in the constant terms obtained by leastsquare regression assuming no uncertainty in the values used to determine the functional form of $\langle E\rangle$. The average prompt gamma ray energy for thermal neutron induced fission of ${ }^{233} \mathrm{U}$ is $0.99 \pm 0.07 \mathrm{MeV}$ using this expression. This value agrees within the uncertainty of the value measured by Pleasonton. ${ }^{6}$ The linear relationship between the average energy of the gamma rays from fission and the atomic mass may only be coincidental or may occur because of differences in the fission yield curves and the subsequent excitation energies of the fission fragments. It is beyond the scope of this work to determine if such a relationship has a physical explanation.

The average number of gamma rays from fission is approximated as

$$
<G>=\frac{E_{t}(\bar{v}, Z, A)}{<E>} .
$$

The expression for $E_{t}(v, Z, A$, $)$ is provided in Eq. 2 and is evaluated using $\bar{v}$ for the fission nuclide. The expression for $\langle E\rangle$ is given in Eq. 4. Application of Eq. 5 for thermal neutron induced fission of ${ }^{233} \mathrm{U}$ yields a value of $6.67 \pm 0.47$ which agrees fairly well with the measured value of $6.31 \pm 0.3$ by Pleasonton. The difference between the estimated average number of prompt gamma rays from fission and Pleasonton's measured value for ${ }^{233} \mathrm{U}$ results because the calculated value of the average energy of prompt fission gamma 
rays from ${ }^{233} \mathrm{U}$ thermal neutron induced fission is underestimated compared to Pleasonton's measured value. These models were used to estimate the properties of prompt gamma rays from the spontaneous fission of various nuclides that are encountered in nuclear safeguard applications. The estimated prompt gamma ray parameters for spontaneous fission of ${ }^{238} \mathrm{U},{ }^{238} \mathrm{Pu},{ }^{240} \mathrm{Pu},{ }^{242} \mathrm{Pu},{ }^{242} \mathrm{Cm}$, and ${ }^{244} \mathrm{Cm}$ are presented in Table 2. The total prompt gamma ray energy was estimated using the average number of neutrons from fission for each nuclide in Eq. 2. The average energy of prompt gamma rays from fission was estimated using Eq. 4, and the average number of prompt gamma rays from fission was estimated using Eq. 5.

Table 2. Estimated properties of gamma rays from spontaneous fission of ${ }^{238} \mathrm{U}$, ${ }^{238} \mathrm{Pu},{ }^{240} \mathrm{Pu},{ }^{242} \mathrm{Pu},{ }^{242} \mathrm{Cm}$, and ${ }^{244} \mathrm{Cm}$ using Eqs. 2, 4, and 5

\begin{tabular}{|c|c|c|c|}
\hline Isotope & $\begin{array}{c}\text { Total energy } \\
(\mathrm{MeV})\end{array}$ & Average number & $\begin{array}{c}\text { Average energy } \\
(\mathrm{MeV})\end{array}$ \\
\hline${ }^{238} \mathrm{U}$ & $6.06 \pm 0.03$ & $6.36 \pm 0.47$ & $0.95 \pm 0.07$ \\
\hline${ }^{238} \mathrm{Pu}$ & $6.14 \pm 0.03$ & $6.34 \pm 0.46$ & $0.97 \pm 0.07$ \\
\hline${ }^{240} \mathrm{Pu}$ & $6.07 \pm 0.03$ & $6.40 \pm 0.47$ & $0.95 \pm 0.07$ \\
\hline${ }^{242} \mathrm{Pu}$ & $6.05 \pm 0.03$ & $6.51 \pm 0.49$ & $0.93 \pm 0.07$ \\
\hline${ }^{242} \mathrm{Cm}$ & $6.26 \pm 0.04$ & $6.62 \pm 0.49$ & $0.95 \pm 0.07$ \\
\hline${ }^{244} \mathrm{Cm}$ & $6.40 \pm 0.04$ & $6.90 \pm 0.52$ & $0.93 \pm 0.07$ \\
\hline
\end{tabular}

\section{MULTIPLICITY DISTRIBUTION OF GAMMA RAYS FROM FISSION}

The multiplicity distribution, $\Pi(G)$, of prompt gamma rays from fission is required for coincidence measurements. Therefore, an approximate method to estimate the multiplicity distribution for spontaneous fission sources is necessary. The multiplicity of prompt gamma rays from the spontaneous fission of ${ }^{252} \mathrm{Cf}$ has been measured by Brunson, ${ }^{17}$ Sokol et al., ${ }^{18}$ Ramamurthy et al., ${ }^{19}$ and Varma et al. ${ }^{20}$ Only Brunson supplied the multiplicity distribution from his measurements. Danilov et al. ${ }^{21}$ performed similar multiplicity measurements for ${ }^{235} \mathrm{U}$ using the measurement technique developed by Ramamurthy et al.

Brunson used a double Poisson model to fit his measured data. The parameters in Brunson's models had no physical significance and represented the best fit of the model to the measured data. Brunson's model for the multiplicity distribution function, $\Pi(G)$, is

$$
\Pi(G)=C_{1} \frac{\left(C_{2}\right) e^{-C_{2}}}{G !}+\left(1-C_{1}\right) \frac{\left(C_{3}\right)^{G} e^{-C_{3}}}{G !},
$$

for $G$ prompt gamma rays from fission. The parameters $C_{1}, C_{2}$, and $C_{3}$ depend on the low-energy gamma ray detection threshold. Brunson's measurements were normalized to 
the average number of ${ }^{252} \mathrm{Cf}$ gamma rays from measurements of Verbinski et al. and Pleasonton et al. The distribution obtained with parameters $C_{1}=0.675, C_{2}=6.78$, and $C_{3}=9.92$ (a $140 \mathrm{keV}$ gamma ray detection threshold) is plotted in Fig. 1. This distribution coincides with the average number of gamma rays from the measurement of Verbinski et al. Brunson developed another distribution that corresponds to the average number of prompt gamma rays from the measurements of Pleasonton et al.

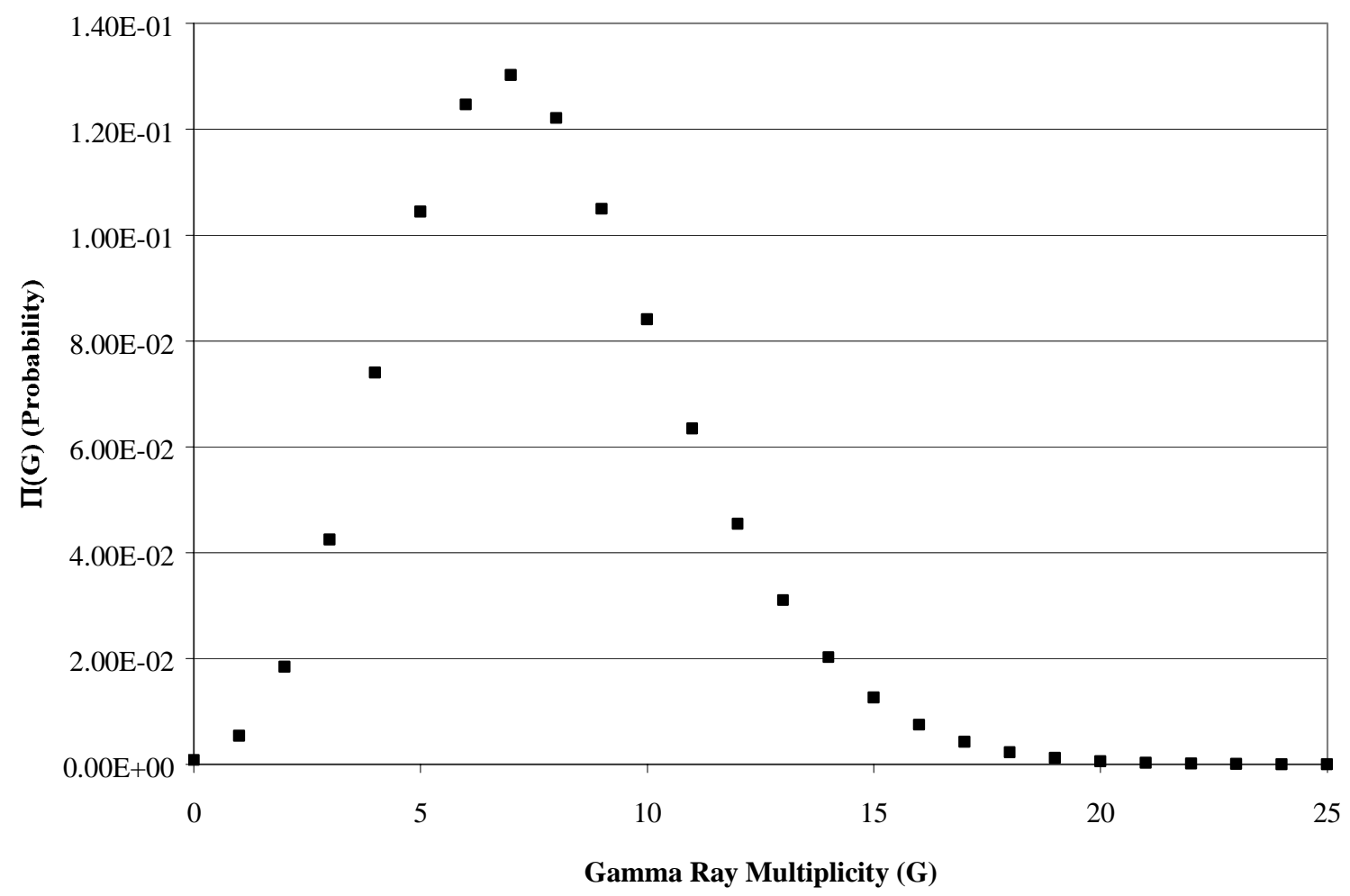

Fig. 1. Gamma ray probability distribution function for spontaneous fission of ${ }^{252}$ Cf from Eq. 6 with parameters $C_{1}=0.675, C_{2}=6.78$, and $C_{3}=9.92$.

The measurements by Sokol et al. produced an average number of prompt gamma rays from fission of 6.58 with a variance of 3.80. This value is much lower than the average number of prompt gamma rays from ${ }^{252} \mathrm{Cf}$ spontaneous fission that was reported by Verbinski et al. and Pleasonton et al. because the low-energy gamma ray detection threshold was $320 \mathrm{keV}$ for measurements of Sokol et al. This high threshold resulted in detection of fewer prompt gamma rays from fission. In addition, the measurements of Sokol et. al. included an upper energy limit of $5120 \mathrm{keV}$. This upper threshold further limits the number of detected gamma rays. Hence, the average of the distribution of Sokol et. al. would be biased too low, and the variance would be underestimated.

The measurements by Ramamurthy et al. assumed an average value of 10.3 prompt gamma rays from fission based on the measurements of Smith et al. ${ }^{16}$ This value is 
inconsistent with more recent measurements. The gamma ray detection threshold and the coincidence time were not specified for this measurement; therefore, it is not possible to determine if either is a significant source of error in these measurements. Moreover, the measurements were performed with only three gamma ray detectors. Three detectors may be too few to obtain meaningful data when the average number is approximately 8 . The average and standard deviation of the number of prompt fission gamma rays from this measurement were 10.3 and $4.2 \pm 0.4$, respectively.

The measurements of Varma et al. were performed in a manner similar to the measurements of Ramamurthy et al. The measurements were performed with only three gamma ray detectors with an $80-\mathrm{keV}$ detection threshold. Again, the number of detectors may be too few to obtain meaningful data. Furthermore, the authors stated that a time-offlight method was used to eliminate the contribution of fission neutron, yet the authors did not provide any indication of the coincidence period for their measurements. The results of Varma et al. were presented as a function of the charge of the fission fragment and are essentially equivalent to the results of Ramamurthy et al.

The measurements by Danilov et al. were performed for thermal neutron induced fission of ${ }^{235} \mathrm{U}$ using the technique developed by Ramamurthy et al. The coincident events among four plastic scintillation detectors were obtained from this measurement. The lower detection threshold and coincidence period were not specified by the authors. The average and standard deviation of the number of prompt fission gamma rays from this measurement were $6.5 \pm 0.3$ and $3.1 \pm 0.1$, respectively.

The measurements of Ramamurthy et al., Varma et al., and Danilov et al. were performed using the same basic principle. The measurements were performed to obtain the average number of counts per fission in two detectors $\left(n_{1}\right.$ and $\left.n_{2}\right)$ and the number of coincident counting events $\left(n_{12}\right)$ between two detectors per fission. If certain assumptions are met, ${ }^{21}$ then the relative width of the distribution of the number of prompt fission gamma rays can be determined. The relative width, $D_{\gamma}$, is defined as

$$
D_{\gamma}=\frac{n_{12}}{n_{1} n_{2}}=\frac{\langle G(G-1)\rangle}{\langle G\rangle^{2}}=\frac{\sigma_{\gamma}^{2}+\bar{G}^{2}-\bar{G}}{\bar{G}^{2}} .
$$

In Eq. 8, $G$ is the number of prompt fission gamma rays, the brackets $<>$ denote an average, $\bar{G}$ is the average number of prompt fission gamma rays, and $\sigma_{\gamma}^{2}$ is the variance of the distribution. By measuring the relative width, the variance of the distribution of the number of prompt fission gamma rays can be determined if the average number of prompt fission gamma rays is known. The relative widths of the distribution of prompt fission gamma rays for spontaneous fission of ${ }^{252} \mathrm{Cf}(1.074 \pm 0.015)^{19}$ and thermal neutron induced fission of ${ }^{235} \mathrm{U}(1.070 \pm 0.006)^{21}$ are essentially the same. The consistency between the two measurements suggests that the relative width of the distribution of the number of prompt fission gamma rays may be independent of the 
fission process. However, the consistency between the measurements of Ramamurthy et al. and Danilov et al. may also result because the measurements were performed using the same experimental method with the same basic assumptions.

Brunson's models depend on three parameters $\left(C_{1}, C_{2}\right.$, and $\left.C_{3}\right)$ that were determined by chi-square minimization between the model and the measured data. Unfortunately, this model cannot be used for estimating the multiplicity distribution of gamma rays for all fission processes because three constraints would be necessary to determine the parameters for Brunson's model. Only estimates or measurements of the average number of prompt fission gamma rays are available from the previous section. However, if the relative width of the distribution of prompt fission gamma rays is assumed to be independent of the fission process, then a two-parameter distribution can be used to describe the distribution of prompt fission gamma rays. The relative width, $D_{\gamma}$, from Brunson's measurement is approximately 1.04. Clearly, neither a binomial distribution nor a Poisson distribution can be used to describe the distribution of prompt fission gamma rays because $D_{\gamma}<1$ for a binomial distribution and $D_{\gamma}=1$ for a Poisson distribution. A negative binomial distribution is one two-parameter distribution with $D_{\gamma}>1$. The negative binomial distribution ${ }^{22}$ is defined as

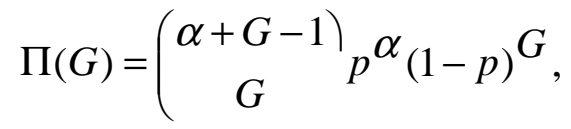

with distribution parameters defined as $p=\bar{x} / \sigma^{2}$ and $\alpha=p \bar{x} /(1-p)$. The parameter $\alpha$ can be redefined in terms of the relative width of the distribution as

$$
\alpha=\left(D_{\gamma}-1\right)^{-1}
$$

Likewise, the parameter $p$ can be redefined as

$$
p=\frac{\alpha}{\alpha+\bar{G}}
$$

A comparison of Brunson's double Poisson distribution with parameters $C_{1}=0.675$, $C_{2}=6.78$, and $C_{3}=9.92$ with the negative binomial distribution for ${ }^{252} \mathrm{Cf}$ prompt fission gamma rays is given in Fig. 2. The negative binomial distribution compares fairly well with Brunson's model. If the parameter $\alpha$ is assumed to be the same for all fission processes, the negative binomial distribution can be used to determine the multiplicity distribution for any fission process given the average number of prompt gamma rays from fission. Thus, the values for the average number of prompt gamma rays from fission that were computed in the previous section can be used to determine the multiplicity distributions by computing the parameter $p$ for each fission process and substituting this value into the equation for the negative binomial distribution. Multiplicity distributions can be produced for ${ }^{238} \mathrm{U},{ }^{238} \mathrm{Pu},{ }^{240} \mathrm{Pu},{ }^{242} \mathrm{Pu},{ }^{242} \mathrm{Cm}$, and ${ }^{244} \mathrm{Cm}$ using the negative binomial distribution. 


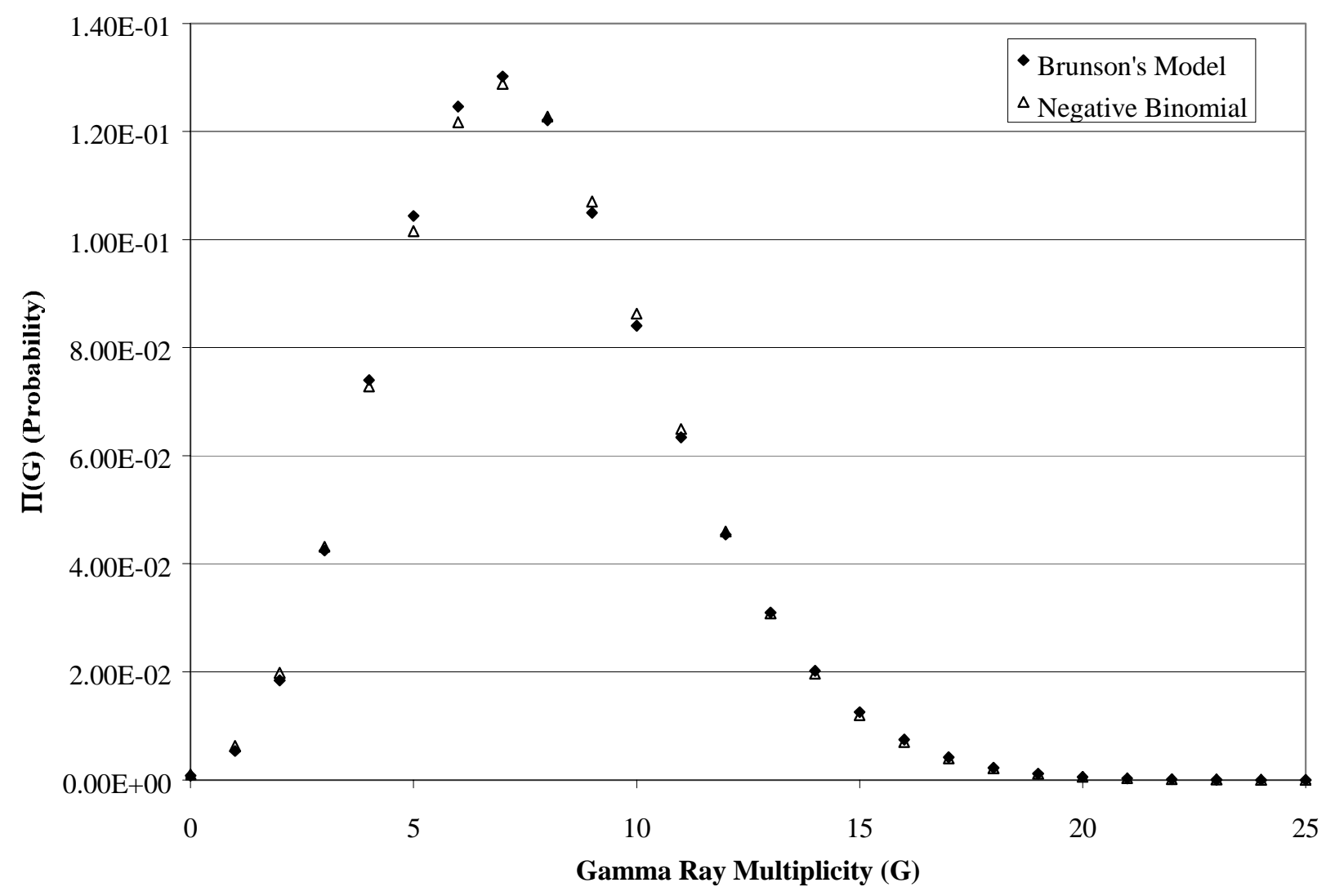

Fig. 2. Comparison of Brunson's model with parameters $C_{1}=0.675, C_{2}=6.78$, and $C_{3}=9.92$ for ${ }^{252} \mathrm{Cf}$ with the negative binomial distribution.

\section{SUMMARY}

Nondestructive assay methods that rely on measurement of correlated gamma rays from fission have been proposed as a means to determine the mass or enrichment of fissile materials. Sensitivity studies for such measurements will require knowledge of the multiplicity of gamma rays from fission; however, a very limited number of multiplicity distributions have been measured. This article has proposed a method to estimate the average number of prompt gamma rays from any fission process by using the correlation of neutron and gamma emission in fission. The models for the total prompt gamma ray energy from fission adequately reproduce the measured value for thermal neutron induced fission of ${ }^{233} \mathrm{U}$. Likewise, the average energy of prompt gamma rays from fission has been adequately estimated using a simple linear model. Additionally, a method to estimate the multiplicity distribution of prompt gamma rays from fission has been proposed based on a measured distribution for ${ }^{252} \mathrm{Cf}$. These methods are only approximate at best and should only be used for sensitivity studies. Measurements of the multiplicity distribution of prompt gamma rays from fission should be performed to determine the adequacy of the models proposed in this article. 
This page intentionally left blank. 


\section{REFERENCES}

1. N. Ensslin, "Principles of Neutron Coincidence Counting," Passive Nondestructive Assay of Nuclear Materials, NUREG/CR-5550 (1991).

2. M. S. Krick and J. E. Swansen, "Neutron Multiplicity and Multiplication Measurements," Nucl. Instrum. Methods, 219, 384-393 (1984).

3. R. B. Perez, J. L. Munoz-Cobo, T. E. Valentine, J. T. Mihalczo, and J. A. Mullens, "Incorporation of Neutron and Gamma Multiplicity Measurements in the ORNL Nuclear Material Identification System (NMIS): Passive and Active Measurements," Proc. INMM Annual Meeting, Phoenix, Arizona, July 1999.

4. J. B. Wilhelmy, E. Cheifetz, R. C. Jared, S. G. Thompson, H. R. Bowman, and J. O. Rasmussen, "Angular Momentum of Primary Products Formed in the Spontaneous Fission of ${ }^{252}$ Cf," Phys. Rev., C5, 2041 (1972).

5. H. Nifenecker, C. Signarbieux, M. Ribrag, J. Poitou, and J. Matuszek, "GammaNeutron Competition in the De-excitation Mechanism of the Fission Fragments of ${ }^{252}$ Cf," Nucl. Phys. A189, 209 (1972).

6. F. Pleasonton, R. L. Ferguson, and H. W. Schmitt, "Prompt Gamma Rays Emitted in the Thermal-Neutron Induced Fission of ${ }^{235}$ U," Phys. Rev., C6, 1023 (1972).

7. F. Pleasonton, "Prompt Gamma Rays Emitted in the Thermal-Neutron Induced Fission of ${ }^{233} \mathrm{U}$ and ${ }^{239} \mathrm{Pu}$," Phys. Rev., A213, 413 (1973).

8. V. V. Verbinski, H. Weber, and R. E. Sund, "Prompt Gamma Rays from ${ }^{235} \mathrm{U}(\mathrm{n}, \mathrm{f}),{ }^{239} \mathrm{Pu}(\mathrm{n}, \mathrm{f})$ and Spontaneous Fission of ${ }^{252} \mathrm{Cf}, "$ Phys. Rev., C7, 1173 (1973).

9. R. W. Pelle and F. C. Maienschein, "Spectrum of Photons Emitted in Coincidence with Fission of ${ }^{235} \mathrm{U}$ by Thermal Neutrons," Phys. Rev., C3, 373 (1971).

10. F. Pleasonton, R. L. Ferguson, and H. W. Schmitt, "Prompt Gamma Rays Emitted in the Thermal-Neutron Induced Fission of ${ }^{233} \mathrm{U},{ }^{235} \mathrm{U}$, and ${ }^{239} \mathrm{Pu}$ and the Spontaneous Fission of ${ }^{252}$ Cf," Oak Ridge National Laboratory, ORNL-4844 (1972).

11. H. R. Bowman and S. G. Thompson, "Prompt Radiation in the Spontaneous Fission of Cf-252," Proc. $2^{\text {nd }}$ International Conference on Peaceful Uses of Atomic Energy (1958), Vol. 15, p. 212, United Nations, Geneva (1958). 
12. E. Nardi, A. Gavron, and Z. Fraenkel, "Total Energy Associated with Prompt Gamma Ray Emission in Spontaneous Fission of ${ }^{252}$ Cf," Phys. Rev., C8, 2293 (1973).

13. G. V. Val'skii, B. M. Aleksandrov, I. A. Baranov, and A. S. Krivokhatskii, G. A. Petrov, and Yu. S. Pleva, "Some Characteristics of the $\gamma$ Radiation Accompanying Fission of ${ }^{252}$ Cf," Soviet Journal of Nucl. Phys., 10, 137 (1969).

14. R. Gwin, R. R. Spencer, and R. W. Ingle, "Measurements of the Energy Dependence of Prompt Neutron Emission from ${ }^{233} \mathrm{U},{ }^{235} \mathrm{U},{ }^{239} \mathrm{Pu}$, and ${ }^{241} \mathrm{Pu}$ for $\mathrm{E}_{\mathrm{n}}=0.005$ to $10 \mathrm{eV}$ Relative to Emission from Spontaneous Fission of ${ }^{252} \mathrm{Cf}$," Nucl. Sci. Eng., 87, 381 (1984).

15. J. W. Boldeman and M. G. Hines, "Prompt Neutron Emission Probabilities Following Spontaneous and Thermal Neutron Fission," Nucl. Sci. Eng., 91, 114 (1985).

16. A. B. Smith, P. R. Fields, and A. M. Friedman, "Prompt Gamma Rays Accompanying the Spontaneous Fission of ${ }^{252}$ Cf," Phys. Rev., 104, 699 (1956).

17. G. Brunson, "Multiplicity and Correlated Energy Gamma Rays Emitted in the Spontaneous Fission of Californium-252," LA-9408-T, Los Alamos National Laboratory (1982).

18. E. A. Sokol, G. M. Ter-Akop'yan, A. I. Krupman, V. P. Katkov, L. F. Nikonova, and N. V. Eremin, "Experiments on the Spontaneous Fission Gamma Photons from ${ }^{248} \mathrm{Cm},{ }^{252,254} \mathrm{Cf},{ }^{256} \mathrm{Fm}$, and ${ }^{259} \mathrm{Md}, "$ Soviet Atomic Energy, 71, 906 (1991).

19. V. S. Ramamurthy, R. K Choudhury, and J. C. Mohan, "Prompt Gamma-Ray Multiplicity Distributions in Spontaneous Fission of Cf-252," Pramana, 8, 322 (1977).

20. R. Varma, G. K. Mehta, R. K. Choudhury, S. S. Kapoor, B. K. Nayak, and V. S. Ramamurthy, "Prompt Gamma-Ray Multiplicity Distributions in Fission of Cf252," Phys. Rev., C43, 1850 (1991).

21. M. M. Danilov, Yu. D. Katarzhnov, V. G. Nedopekin, and V. I. Rogov, "Investigation of the Distribution in the Number of Prompt Gamma Rays and Their Correlations with Prompt Neutrons in Fission of ${ }^{235} \mathrm{U}$," Soviet Journal of Nucl. Phys., 47, 972 (1988).

22. J. E. Freund, Mathematical Statistics, Prentice-Hall, Inc., Englewood Cliffs, New Jersey, 1962. 


\title{
DISTRIBUTION
}

\author{
L. G. Chiang \\ K. H. Guber \\ P. E. Koehler \\ L. C. Leal \\ J. A. March-Leuba \\ J. K. Mattingly \\ J. T. Mihalczo \\ R. B. Oberer \\ R. B. Perez \\ R. W. Roussin \\ T. E. Valentine (20) \\ J. D. White \\ M. C. Wright \\ ORNL Laboratory Records - RC \\ ORNL Laboratory Records, OSTI
}

